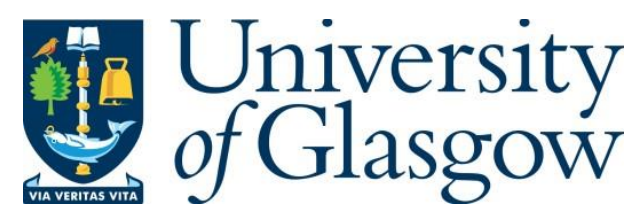

Wang, J., Yu, X., Wang, C., Xiang, K., Deng, M. and Yin, H. (2017) PAMPS/MMT composite hydrogel electrolyte for solid-state supercapacitors. Journal of Alloys and Compounds, 709, pp. 596-601.

There may be differences between this version and the published version. You are advised to consult the publisher's version if you wish to cite from it.

http://eprints.gla.ac.uk/141880/

Deposited on: 19 July 2017

Enlighten - Research publications by members of the University of Glasgow http://eprints.gla.ac.uk 


\title{
PAMPS/MMT composite hydrogel electrolyte for solid-state supercapacitors
}

\author{
Jin Wang*a ${ }^{\mathrm{a}}$, Xianghu Yu ${ }^{\mathrm{a}}$, Cui Wang ${ }^{\mathrm{a}}$, Kechuang Xiang ${ }^{\mathrm{a}}$, Mengde Deng ${ }^{\mathrm{a}}$, Huabing Yin ${ }^{\mathrm{b}}$ \\ ${ }^{a}$ School of Chemistry and Chemical engineering, Hefei University of Technology, Hefei 23009, P. R. \\ China \\ ${ }^{\mathrm{b}}$ Department of Electronics and Electrical Engineering, University of Glasgow, G12 8QQ, UK \\ *Corresponding author Tel.: +86 551 62905660; fax: +86 55162905660. \\ E-mail address: jinwang@hfut.edu.cn (Jin Wang).
}

ABSTRACT: PAMPS/MMT composite electrolytes with high ionic conductivities were prepared by solution-casting method. The influences of Na-MMT contents on the ionic conductivities and mechanical properties of PAMPS hydrogel electrolyte were characterized and the electrochemical properties of supercapacitor with PAMPS/MMT composite electrolytes were investigated. The addition of Na-MMT can increase the ionic conductivities, tensile strength of PAMPS. PAMPS/MMT composite electrolyte has the superior integrated performance of ionic conductivities and tensile strength when the mass fraction of Na-MMT is about $5 \sim 7 \%$, the ionic conductivity is $5.91 \times 10^{-2} \mathrm{~S} \mathrm{~cm}^{-1}$ and the tensile strength is $8.9 \mathrm{MPa}$. The capacitor exhibits ideal behavior for the electronic double-layer capacitance. The specific capacitance and the cycling stability of PAMPS/MMT composite electrolytes are enhanced in comparison with that of PAMPS. Thus it provides a promising composite polyelectrolyte for supercapacitor.

Keywords: hydrogel electrolyte, composite, ionic conductivity, supercapacitors 


\section{Introduction}

Solid-polymer electrolytes with the advantages of compactness, reliability and freedom from leakage have been used widely for different electrochemical devices, such as rechargeable batteries, electrochromic displays and supercapacitors ${ }^{[1-3]}$. They can be classified into dry solid-polymer electrolytes, gel-polymer electrolytes and composite-polymer electrolytes. Gel-polymer electrolytes (GPEs) always exhibit high conductivity than that of dry solid-polymer electrolytes. Various polymers ${ }^{[4-8]}$, such as poly(ethylene oxide) (PEO), poly(vinyl alcohol) (PVA), poly(acrylonitrile), Poly(ethylene glycol) (PEG), poly(vinylidene fluoride) (PVDF) and so on, have been reported to form GPEs with conductivities ranging between $10^{-4}$ and $10^{-3} \mathrm{~S} \mathrm{~cm}^{-1}$ under ambient conditions. GPEs have high swell capacity of ionic or salt solutions to improve their ionic conductivities, and also have good mechanical properties such as flexibility and stretchability. Which has arousing the interest in flexible and wearable energy devices for application in flexible and stretchable electronics, and have been reported not only could be useful for fully wearable energy storage devices, but also have high efficient performance ${ }^{[9-11]}$. GPEs are generally prepared using organic solvents. In contrast, aqueous GPEs have a better application prospect for the cost and environmental security to consider, although those commercial supercapacitors utilize non-aqueous organic electrolytes can reach cell voltage as high as $3 \mathrm{~V}^{[12-13]}$.

Hydrogels are three-dimensional polymeric networks which due to the presence of chemical cross-linkages or physical entanglements with corresponding water absorption capacity. Hydrogels can be classified as neutral or ionic, including anionic, 
cationic and zwitterionic, based on the nature of the pendant groups in the precursor. The neutral polymer electrolytes include PEO, PVA and PEG can be leveraged as matrix for solid polymer electrolyte because of their good film formability and easily be changed into gel or hydrogel. The neutral GPEs such as PEO obtain higher conductivity values in solvent-free polymer electrolytes only when the polymer is in the amorphous state, when the temperature is higher than $70^{\circ} \mathrm{C}$, i.e., the melting point of PEO ${ }^{[14]}$. The ion conductivities of the neutral GPEs can also be increased at ambient temperature by blending with ionic polymer, exactly as in PVA matrix mixed with poly (acrylic acid) ${ }^{[15]}$. Except for acrylic acid, the ionic monomers with amino, sulfonate, cyano group and the others can be polymerized into ionic polymer with higher ionic conductivities ${ }^{[16-18]} .2$-acrylamide-2-methyl propanesulfonic acid (AMPS) is an amphiphilic compound in which the sulfonic acid groups make it water-soluble and salt resistant, amide groups make it thermal-stable and hydrolysis resistant. Polymer polymerized from AMPS (PAMPS) can obtained higher conductivity based on the ionic molecular structure in comparison with the neutral GPEs. PAMPS has being blended or grafted with the other polyelectrolyte in fuel cell for its advantage on ion transportation, water absorption and retention capacity ${ }^{[19-20]}$.

Hydrogels properties such as mechanical, thermal, electrical properties and responsiveness can be improved by preparing a composite polyelectrolyte from inorganic material and polymer. The addition of inorganic material improves the proportion of amorphous region in polymer, helps chain segments move easily, meanwhile, increases the interface stability between polyelectrolyte and electrode. 
The composites from polymer and clays have been researched widely ${ }^{[21]}$, of which sodium montmorillonite (Na-MMT) shows a better performance in water-absorption, ion-exchange and water-dispersive capacities. As far as the application of supercapacitor is concerned, electrolytes affect the capacitance, operation voltage, energy density and power density. Electrolytes with higher ion conductivity and good mechanical properties are worth developing. Here, the PAMPS/MMT composite hydrogels were prepared, and the influences of Na-MMT on the tensile strengh, ionic conductivities of PAMPS were investigated. They are potential candidates as a component for electrochemical capacitors, and the performances of the PAMPS/MMT composite were explored when used for GPEs of supercapacitors.

\section{Experimental}

\subsection{Material}

2-Acrylamido-2-methyl-1-propanesulfonic acid (AMPS) (Aladdin, 98\%) and N'Nmethylene-bis-acrylamide (MBA) (Aladdin, 97\%) were used as received. Potassium persulfate (KPS) (Aldrich, 98\%) was used after recrystallized. Potassium hydroxide $(\mathrm{KOH})(96 \%$, Sinopharm Chemical Reagent Co. Ltd) used without any purification. Na-montmorillonite (Na-MMT) (95\%) was sourced from Fenghong Co.. Activated carbon, acetylene black, PVDF powder and N-methylpyrrolidone (NMP) were purchased from Sinopharm Chemical Reagent Co. Ltd., deionized water was used in all reactions.

\subsection{Synthesis of the PAMPS/MMT composite hydrogel}

A series of PAMPS/MMT composite electrolytes with different weight contents of 
Na-MMT were prepared by solution-casting method. Dissolve $3 \mathrm{~g}$ AMPS in $20 \mathrm{~mL}$ deionized water and pour them into a reactor, and then Na-MMT was put into the solution for ultrasonic $24 \mathrm{~h}$ to well disperse the clay particles. Subsequently, the solution with a certain amount of MBA crosslinker (3\% in weight) and KPS initiator ( $0.4 \%$ in weight $)$ were added to the reactor while stirring. The determination of the amount of MBA and KPS is seen details in Supplementary Information. Afterwards, the homogeneous liquid mixtures were poured into a PTFE mould at a vacuum drying oven for desiccation ( $12 \mathrm{~h}$ at 70 ). The schematic representation of the synthesis technique of PAMPS/MMT composite is illustrated in Fig.1. PAMPS hydrogel was also prepared with the above method for comparison.

\subsection{Fabrication of the electrochemical capacitors (EC)}

An electrochemical capacitor was fabricated by sandwiching the GPE between the two electrodes as shown in Fig. 2. The electrodes used in ECs are self-made activated carbon electrodes. Activated carbon was grinded into powder, mix activated carbon, acetylene black and PVDF binder (8:1:1 by weight), then a required amount of NMP was added into the mixed powder dropwise with constant stirring to form slurry, then the slurry was agitated in an ultrasonic water bath for 30 min for a better dispersion. After that, the slurry was taken out and coated on the porous nickel sheets uniformly which used as current collectors, leave the sheets in vacuum drying oven in 70 for $24 \mathrm{~h}$ to get the final activated carbon electrodes. PAMPS/MMT composite hydrogel simultaneously served as separator and electrolyte, and was sandwiched between two symmetrical self-made activated carbon electrodes, the composite electrolyte used 
must be soaked in $4 \mathrm{~mol} / \mathrm{L} \mathrm{KOH}$ solution till its weight keeps unchanged, which means $\mathrm{KOH}$ solution entrapped in polyelectrolyte is saturated, the swelling capacity $\left(W_{\mathrm{KOH}} / \mathrm{g} \mathrm{g}^{-1}\right)$ is $8 \mathrm{wt} \%$ and calculated according to the equation:

$$
W_{K O H}=\frac{W_{2}-W_{1}}{W_{1}} \times 100 \%
$$

where $W_{1}$ is the weight of the polyelectrolyte which was dried in vacuum drying oven in 70 till its weight gets unchangeable, while $W_{2}$ is the weight of the polyelectrolyte which was immersed in 4mol/L KOH solution till its weight gets unchangeable.

\subsection{Characterization}

X-ray diffraction (XRD) curves were recorded using a Rigaku D/Max-2550 diffractometer with $\mathrm{Cu}-\mathrm{K} \alpha$ radiation $(\lambda=1.5418 \AA)$. Fourier transform infrared spectroscopy (FT-IR) was performed on a FT-IR spectrometer (PE 1650). The thermal stability of PAMPS/MMT composite hydrogel was characterized using a thermogravimetric analysis (TGA) (TG 209 F3 Netzsch, Germany) with a heating rate of $10 /$ min under $\mathrm{N}_{2}$ atmosphere. Tensile test was performed with a CMT4104 electronic universal tester (MTS Ind. Co., China), and stretched at a constant velocity of $10 \mathrm{~mm} / \mathrm{min}$.

The measurement of ionic conductivity of the PAMPS/MMT composite hydrogel is carried out by electrochemical impedance spectroscopy (EIS) using CHI660B electrochemical workstation. The GPEs was sandwiched by two stainless steels with the mode of SS/PAMPS/MMT/SS (SS notes stainless steels). The bulk ionic conductivity $(\sigma)$ of the PAMPS/MMT composite electrolyte were determined from the complex impedance spectra in the frequency range between $0.01 \mathrm{~Hz}$ and $100 \mathrm{kHz}$ 
with a perturbation of $5 \mathrm{mV}$ rms using the equation ${ }^{[22]}$

$$
\sigma=\frac{L}{R_{b} \cdot A}
$$

where L, A and $R_{\mathrm{b}}$ are the thickness, area, and bulk resistance (calculated from high-frequency intercept on the real impedance axis of the Cole-Cole plot), respectively.

The cyclic voltammograms $(\mathrm{CV})$ were carried out with different scan rates using a two-electrode configuration. Life-cycle tests were performed with galvanostatic charge-discharge measurements (GCD), the specific capacitance (C) of the ECs was calculated from the discharge curves of the charge-discharge plots using the equation [23].

$$
C=\frac{I \Delta t}{m \Delta V}
$$

where $I$ is the constant current, $\Delta t$ is the time required to change the potential by $\Delta V$, $\Delta V$ is the potential difference, and $m$ is the weight of activated carbon in the electrode, respectively.

\section{Results and Discussion}

\subsection{Structure of PAMPS/MMT composite hydrogel}

Fig. 3 recorded infrared spectra of pristine Na-MMT, PAMPS and PAMPS/MMT composite, respectively. From the FT-IR spectrum of PAMPS/MMT, it can be shown that the absorption peaks at $2960 \sim 2850 \mathrm{~cm}^{-1}$ are related to $\mathrm{C}-\mathrm{H}$ stretching of PAMPS molecules, the peaks at $1646 \mathrm{~cm}^{-1}$ and $1559 \mathrm{~cm}^{-1}$ are attributed to the bending of $\mathrm{NH}$ bonds and the peak at $1372 \mathrm{~cm}^{-1}$ is related to $\mathrm{S}=\mathrm{O}$ of PAMPS, respectively. The peaks 
at $1036 \mathrm{~cm}^{-1}$ and $461 \mathrm{~cm}^{-1}$ are attributed to the stretching and the bending of Si-O band, respectively. The peak at $522 \mathrm{~cm}^{-1}$ is assigned to the Si-O-Al bending vibration of the silicate layers ${ }^{[24]}$, which indicates that the existence of MMT clay layers although somewhat overlapping between bands originating from both clay and PAMPS. On comparing the FTIR spectra of MMT, the intensities of peaks due to $\mathrm{Si}-\mathrm{O}$ and $\mathrm{Al}-\mathrm{O}$ of $\mathrm{MMT}$ are reduced for its small addition in polymer matrix. The composites were analyzed by X-ray diffraction to obtain the structure of the clay in the polymer matrix. Fig.4 exhibits the XRD patterns of Na-MMT, PAMPS/MMT composite hydrogel with 3\% MMT, 5\% MMT, 7\% MMT and 9\% MMT, respectively. As shown from the XRD pattern of Na-MMT, the diffraction peaks of Na-MMT are at $7.0^{\circ}, 20.0^{\circ}, 26.7^{\circ}, 28.4^{\circ}, 35.0^{\circ}, 42.3^{\circ}$ and $54.4^{\circ}$, respectively ${ }^{[25]}$. These characteristic peaks are disappeared after composited with PAMPS just a broad peak at ca. $15^{\circ} \sim 30^{\circ}$, which indicates that the weak crystallization of PAMPS/MMT composite and laminar structure of MMT has been disappeared. The XRD pattern of pure Na-MMT shows a strong peak at $2 \theta=7.0^{\circ}$ which corresponds to an interlayer space of $d=12.6 \AA$. In the XRD patterns of PAMPS/MMT composite with 3\% and 5\% MMT, no peak appears at $2 \theta=7.0^{\circ}$ indicating that MMT layer has been exfoliated in hydrogel matrix. In the XRD patterns of PAMPS/MMT composite with $7 \%$ and $9 \%$ MMT, a small peak at $2 \theta=7.0^{\circ}$ indicating that not all the MMT were intercalated and the nocomposite with partially exfoliated structures were obtained. As it can be observed, clay has a better dispersion in polymer matrix when a small addition was employed, and the agglomeration occur always at a larger addition of MMT. MMT is 
an expandable clay mineral and has colloidal activity. The clay layers are held together by a relative weak intermolecular force, and water or hydrophilic molecules easily penetrate into the interlayer region.

\subsection{Mechanical properties of PAMPS/MMT composite hydrogel}

The relationship between content of Na-MMT and tensile strength of the PAMPS/MMT composite hydrogels were studied. According to the data in Table 1, the tensile strength of PAMPS/MMT composite hydrogels increased with the increasing amount of Na-MMT firstly, the tensile strength reached the maximum value of $8.9 \mathrm{MPa}$ when the content of Na-MMT is ca. $7 \%$, which is higher than that of PAMPS hydrogel with the value 6.9 MPa. This should be related with the addition of Na-MMT. The small addition is favor of Na-MMT dispersed in PAMPS. MMT layers were exfoliated into the hydrogel homogeneously according the analysis of XRD. The well-dispersed inorganic particles in polymer can also act as physical cross-linking points. In other word, the cross-linking density of the composite hydrogels increased and obtains the higher mechanical properties. The tensile strength of PAMPS/MMT composite hydrogels decreased when loading increased, and the value at $11 \%$ is even lower than that of PAMPS hydrogel. This may be related with the agglomeration of Na-MMT in PAMPS hydrogel. The inorganic particles are gathered easily to form aggregation for too close each other when add a large amount of Na-MMT. These aggregations act as the point of stress concentration when stretching the hydrogel and make the tensile strength decreased.

\subsection{Thermal properties of PAMPS/MMT composite hydrogel}


Thermal stability and decomposition behavior of the PAMPS and PAMPS/MMT composite hydrogel were characterized through TGA. As shown in Fig.5, which indicate a two-step degradation pattern for both hydrogels. The weight loss around 220 may be ascribed to the decomposition of the sulfonate groups, amino groups and the other side chains of PAMPS molecule. The second degradation step starts at about 300 is mainly attributed to degradation of cross-linking bridge and polymer backbone. In the first stage of $200 \sim 300$, PAMPS/MMT hydrogel exhibits the higher thermal stability than that of PAMPS, which should be ascribed to the interreaction between Na-MMT layer and the ionic groups of PAMPS. In the second stage of $300 \sim 500$, the same thermal stabilities occur in both hydrogels, which should be ascribed to the combined action of cross-linking and crystallization of PAMPS molucule. The addition of Na-MMT results in higher cross-linking density and increasing the thermal stability, meanwhile, the crystallinity of PAMPS decreased when the degree of cross-linking density increased, which may weaken the thermal stability.

\subsection{Conductivity of PAMPS/MMT composite hydrogel}

As basic and important element of polyelectrolyte used in electrochemical capacitors, ionic conductivity of the PAMPS/MMT GPEs were examined and the influenced of the addition of Na-MMT were studied. Fig.6 (a) is the electrochemical impedance spectra of with different proportions of PAMPS/MMT composite electrolyte, and Fig.6 (b) is the enlarged part of the impedance response at high frequency region. From the enlarged figure, it can be found that the influence of 
Na-MMT on PAMPS/MMT composite electrolyte has a similar trend with that of tensile strength. The data has been listed in Table 2. Firstly, the ionic conductivity increases with increasing the amount of Na-MMT and the maximum value reaches up to $5.91 \times 10^{-2} \mathrm{~S} \mathrm{~cm}^{-1}$ when the mass fraction is $5 \%$, and then the ionic conductivity of PAMPS/MMT composite electrolyte began to decrease with increasing Na-MMT continually. The improvement of ionic conductivity in PAMPS/MMT composite electrolyte represents good current supply for the supercapacitors, and it can be explained mainly in two aspects. The one is the improvement of tensile strength can enhance the interface stability between polyelectrolyte and electrode. Inorganic MMT particles dispersed well in PAMPS matrix increased the degree of physical cross-linking. The other is the inorganic MMT particles can increase the size of amorphous region of PAMPS and create the hole of ion transportation in the area of particle surface ${ }^{[26-27]}$. PAMPS embedded into Na-MMT lamellae and decreased the degree of crystallinity of polymer when the content of Na-MMT is relatively low, which is in favor of ion movement in polyelectrolyte. Meanwhile, Na-MMT has good ion-exchange capability which is also a contribution to ionic conductivity. The ionic conductivities decreased when Na-MMT loading increased, it should be attributed to the lack of interactions between the inorganic particles and the polymer for the sake of the crude dispersion. High ionic conductivity at room temperature and good mechanical properties or dimensional stability is the required fundamental performance of polyelectrolyte when used in electrochemical supercapacitor ${ }^{[28]}$. According the research above, we can think that the superior combination properties 
of PAMPS/MMT GPEs can be obtained are in the range of 5 7\% with Na-MMT.

\subsection{Electrochemical performance of the PAMPS/MMT GPEs}

$\mathrm{CV}$ was studied with a potential range of $0 \sim 1 \mathrm{~V}$ at the scan rate of $20 \mathrm{mV} / \mathrm{s}$. As seen in Fig.7 (a), there are no peaks caused by redox reactions in both GPEs, the shape of $\mathrm{CV}$ plots is close to a rectangular one, which suggests a nearly ideal behavior for the electronic double layer capacitor (EDLC). The resulting rectangle areas from PAMPS/MMT composite electrolyte is larger than that from PAMPS electrolyte, which reveals that specific capacitance value of activated carbon-based supercapacitors applying PAMPS/MMT GPE is higher than that of PAMPS GPE. The specific capacitance $(\mathrm{C})$ from $\mathrm{CV}$ plots is defined by this equation:

$$
C=\frac{2 S}{m v \Delta V}
$$

where $S$ is the rectangle area, $v$ is the scan rate, $m$ is the weight of activated carbon in the electrodes, $\Delta V$ is the potential difference, respectively. The specific capacitance of PAMPS/MMT GPE based supercapacitor calculated by the equation (4) is $22 \mathrm{~F} \mathrm{~g}^{-1}$, which is higher than $20 \mathrm{~F} \mathrm{~g}^{-1}$ of the PAMPS GPE based supercapacitor. Considering that there is no pseudocapacitive material in electrode, the capacitance enhancement is mainly due to the better EDLC's behaviour, which means the PAMPS/MMT composite electrolyte is superior to the PAMPS polyelectrolyte being applied to EC. CV plots of EC assembled with PAMPS/MMT GPEs at different scan rates is presented in Fig. 7 (b). With the increase of scan rate, the CVs kept the shape of a rectangular and the current value is found to increase with increasing the scan rate, which is in accordance with the equation $I=C d V / d t$ of EDLC. The currents (I) are 
very stable and unchanged through cycling, which indicates that the ionic in the solution entrapped in the polymer matrix transports smoothly. Fig.8 (a) shows the galvanostatic charge-discharge curves for the EC with the PAMPS, PAMPS/MMT composite polyelectrolyte and $4 \mathrm{M} \mathrm{KOH}$ aqueous solution electrolyte at a current density of $10 \mathrm{~mA} \mathrm{~g}^{-1}$, respectively. Almost linear charge and discharge curves were observed, which corresponds to those of the ideal EDLC. It is also indicated that good electrode/electrolyte interface was formed in each experimental EC and that the experimental EC successfully worked as an EDLC. Moreover, the curves for all the three electrolytes were very similar, probably due to the high ionic conductivity and good contact with the electrode of the polyelectrolytes as well as the $\mathrm{KOH}$ aqueous solution electrolyte. The specific capacitance was calculated from the discharge curve of 4M KOH aqueous solution electrolytes, PAMPS/MMT composite electrolyte and PAMPS electrolyte are 25,22 and $20 \mathrm{~F} \mathrm{~g}^{-1}$, respectively. The low values of the capacitances may be for the imperfection of the self-made activated carbon electrode. However, we still find that the capacitance of PAMPS/MMT GPE was closed to that of $4 \mathrm{M} \mathrm{KOH}$ aqueous solution electrolyte, which implies the good transportation of the ion entrapped in PAMPS/MMT GPE. Comparing with PAMPS GPE, the higher capacitance of the composite polyelectrolyte means Na-MMT doped in PAMPS does in favor of improving ion transportation. Cycling performance is another critically important characteristic for evaluating the stability of EC. The discharging cycles at a current density of $0.2 \mathrm{~A} \mathrm{~g}^{-1}$ were tested. As seen from Fig.8 (b), the PAMPS/MMT GPE based EC exhibits a cycling stability of $90 \%$ retention up to 1000 cycles, which 
is better than $70 \%$ retention of PAMPS GPE based EC. This result suggests that the PAMPS/MMT GPE is more stable under charge-discharge cycles and keeping good interfacial contact with the activated carbon electrodes.

\section{Conclusions}

PAMPS/MMT composite hydrogel with high ionic conductivities were prepared successfully in the present of MBA cross-linker and KPS initiator. With $\mathrm{KOH}$ solution as the conducting salt, the electrochemical properties of supercapacitor with PAMPS/MMT as polyelectrolyte were also explored. The addition of Na-MMT can increase the ionic conductivities, tensile strength of PAMPS. When the content of Na-MMT is lower, MMT layers were exfoliated into PAMPS hydrogel homogeneously and the well-dispersed inorganic particles in polymer matrix can act as physical cross-linking points to make the higher mechanical properties. Meanwhile, the degree of crystallinity of PAMPS decreased with increasing the cross-linking, which is in favor of ion movement in polyelectrolyte and therefore the higher ionic conductivity was obtained. The MMT particles easy to form aggregation for too close each other when load increasing. These aggregates act as the point of stress concentration when stretching the hydrogel and make the tensile strength decreased. The same trend occurred in the influence of Na-MMT on the ionic conductivities of PAMPS/MMT for these aggregates hampered the ion transportation in the polyelectrolyte. The capacitor applying PAMPS and PAMPS/MMT GPE as electrolytes exhibits ideal behavior for the electronic double-layer capacitance. The specific capacitance and the cycling stability of PAMPS/MMT composite electrolytes 
are enhanced in comparison with that of PAMPS. The capacitor performances are close related to the fabrication of the capacitor device including the electrode materials. PAMPS/MMT GPE has exhibited the properties in mechanical strength, ionic conductivity and thermal stability which are meeting with applied in solid supercapacitor. It should be a promising GPE for supercapacitor if further optimizing design of the device.

\section{References}

[1] C. Zhong, Y. Deng, W. Hu, J. Qiao, L. Zhang, J. Zhang, Chem. Soc. Rev. 44 (2015) 7484-7539.

[2] E.M. Masoud, A.A. El-Bellihi, W.A. Bayoumy, M.A. Mousa, J. Alloys Compd. 575 (2013) 223-228.

[3] M.L. Verma, M. Minakshi, N.K. Singh, Electrochim. Acta 137 (2014) 497-503.

[4] A.S. Westover, F.N. Shabab, J.W. Tian, S. Bernath, L. Oakes, W.R. Erwin, C.L. Pint, Electrochem. Soc. 161 (2014) E112-E117.

[5] C.S. Lim, K.H. Teoh, C.W. Liew, S. Ramesh, Mater. Chem. Phys. 143 (2014) 661-667.

[6] P.C. Lee, T.H. Han, T. Hwang, J.S. Oh, S.J. Kim, B.W. Kim, J.D. Nam, J. Membr. Sci. 409 (2012) 365-370.

[7] N.E.A. Shuhaimi, L.P. Teo, H.J. Woo, S.R. Majid, A.K. Arof, Polym. Bull. 69 (2012) 807-826.

[8] M.K. Singh, M. Suleman, Y. Kumar, S.A. Hashmi, Energy 80 (2015) 465-473.

[9] L.B. Dong, C.J. Xu, Y. Li, Z.H. Huang, F.Y. Kang, Q.H. Yang, X. Zhao, J, Mater. Chem. A 4 (2016) 4659-4685. 
[10] J. Bae, M.K. Song, Y.J. Park, J.M. Kim, M. Liu, Z.L. Wang, Angew. Chem. Int. Ed. 50 (2011) $1683-1687$.

[11] L. Dong, C. Xu, Y. Li, Z. Pan, G. Liang, E. Zhou, F. Kang, Q.H. Yang, Adv. Mater. 28 (2016) 9313-9319.

[12] N.A. Choudhury, S. Sampath, A.K. Shukla, Energy Environ. Sci. 2 (2009) 55-67.

[13] A.A. Łatoszyńska, G.Z. Żukowska, I.A. Rutkowska, P.L. Taberna, P. Simon, P.J. Kulesza, W. Wieczorek, J. Power Sources 274 (2015) 1147-1154.

[14] S. Ketabi, K. Lian, Solid State Ionics 227 (2012) 86-90.

[15] Y.E. Miao, J. Yan, Y. Huang, W. Fan, T. Liu, RSC Adv. 5 (2015) 26189-26196.

[16] J. Pan, Y. Li, L. Zhuang, J. Lu, Chem. Commun. 46 (2010) 8597-8599.

[17] V.K. Sachan, A. Devi, R.S. Katiyar, R.K. Nagarale, P.K. Bhattacharya, Eur. Polym. J. 56 (2014) 45-58.

[18] X. Peng, H. Liu, Q. Yin, J. Wu, P. Chen, G. Zhang, Y. Xie, Nat. Commun. 7 (2016) 2-8.

[19] N.W. Deluca, Y.A. Elabd, J. Polym. Sci. Part B: Polym. Phys. 44 (2006) 2201-2225.

[20] L. Chikh, V. Delhorbe, O. Fichet, J. Membr. Sci. 368 (2011) 1-17.

[21] K. Kabiri, H. Omidian, M.J. Zohuriaan - Mehr, S. Doroudiani, Polym. Compos. 32 (2011) 277-289.

[22] N.A. Choudhury, A.K. Shukla, S. Sampath, S. Pitchumani, J. Electrochem. Soc. 153 (2006) A614-A620.

[23] G.P. Pandey, S.A. Hashmi, Electrochim. Acta 105 (2013) 333-341.

[24] N. Greesh, P.C. Hartmann, V. Cloete, R.D. Sanderson, J. Colloid Interface Sci. 319 (2008) $2-11$. 
[25] L. Zhou, H. Chen, X. Jiang, F. Lu, Y. Zhou, W. Yin, X. Ji, J. Colloid Interface Sci. 332 (2009) $16-21$.

[26] M.F. Samberan, M.M. Hasani-Sadrabadi, S.R. Ghaffarian, A. Alimadadi, Int. J. Hydrogen Energy 38 (2013) 14076-14084.

[27] M.M. Hasani-Sadrabadi, E. Dashtimoghadam, F.S. Majedi, K. Kabiri, M. Solati-Hashjin, H. Moaddel, J. Membr. Sci. 365 (2010) 286-293.

[28] X. Lu, M. Yu, G. Wang, Y. Tong, Y. Li, Energy Environ. Sci. 7 (2014) 2160-2181. 


\section{Captions for Figures}

Fig.1 Schematic presentation of the experimental method

Fig.2 Schematic presentation for the EC model

Fig.3 FT-IR spectra of (a) PAMPS, (b) PAMPS/MMT composite hydrogel and (c)Na-MMT

Fig.4 XRD patterns of PAMPS/MMT composite hydrogel with different proportion of Na-MMT

Fig.5 TGA thermograms of PAMPS and PAMPS/MMT composite hydrogel

Fig.6 (a) EIS of PAMPS/MMT GPEs, (b) the partial enlarge plots of EIS

Fig.7 (a) CV plots of the EC based on PAMPS and PAMPS/MMT GPE, (b) CV plots of the EC based on PAMPS/MMT GPE at different scan rates

Fig.8 (a) Galvanostatic charge-discharge curves of the EC with (1) PAMPS GPE, (2)PAMPS/MMT GPE and (3) 4M KOH aqueous solution electrolyte; (b) cycling performance of ECs based on PAMPS and PAMPS/MMT GPE at a current density of $0.2 \mathrm{~A} \mathrm{~g}^{-1}$

\section{Captions for Tables}

Table 1 Tensile strength of the PAMPS/MMT composite hydrogel with different proportion of

Na-MMT

Table 2 Ionic conductivity of the PAMPS/MMT composite electrolyte with different proportion of Na-MMT 


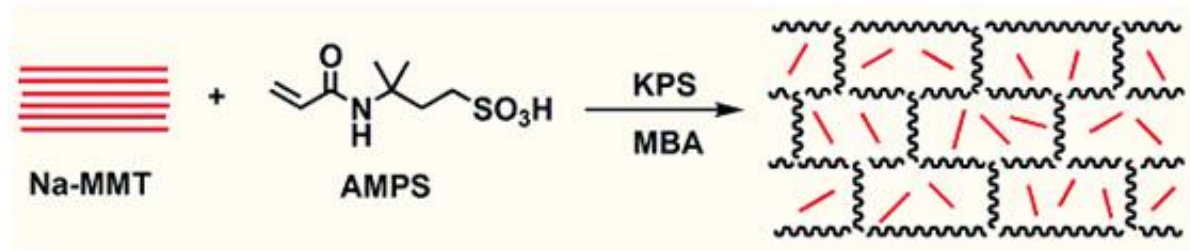

Fig.1 Schematic presentation of the experimental method

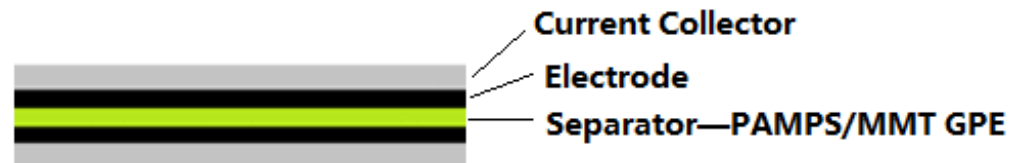

Fig.2 Schematic presentation for the EC model

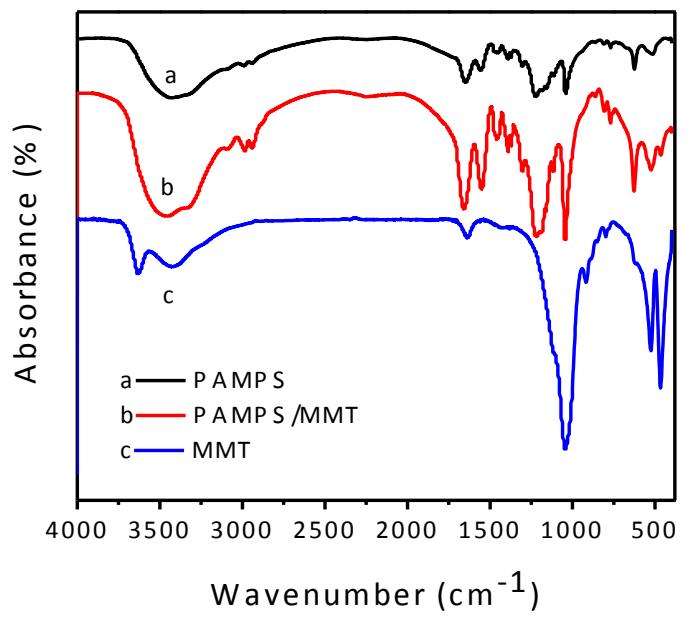

Fig.3 FT-IR spectra of (a) PAMPS, (b) PAMPS/MMT composite hydrogel and (c)Na-MMT 


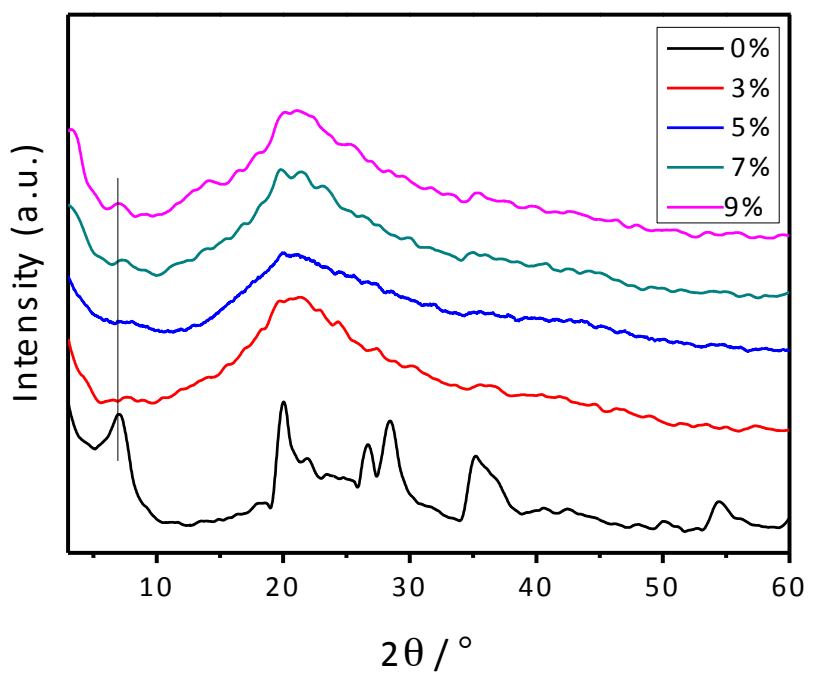

Fig.4 XRD patterns of PAMPS/MMT composite hydrogel with different proportion of Na-MMT

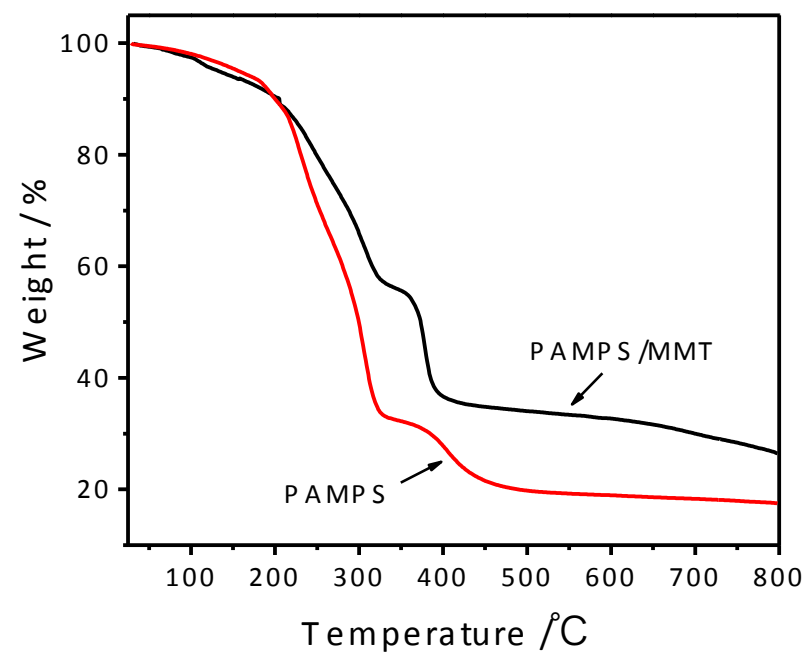

Fig.5 TGA thermograms of PAMPS and PAMPS/MMT composite hydrogel
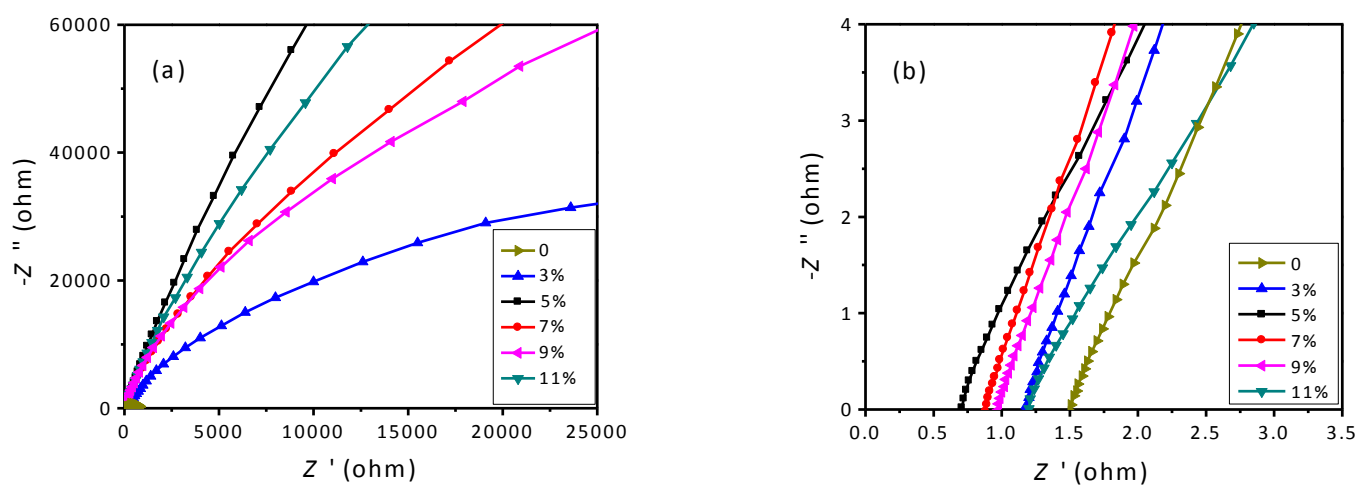

Fig.6 (a) EIS of PAMPS/MMT GPEs, (b) the partial enlarge plots of EIS 

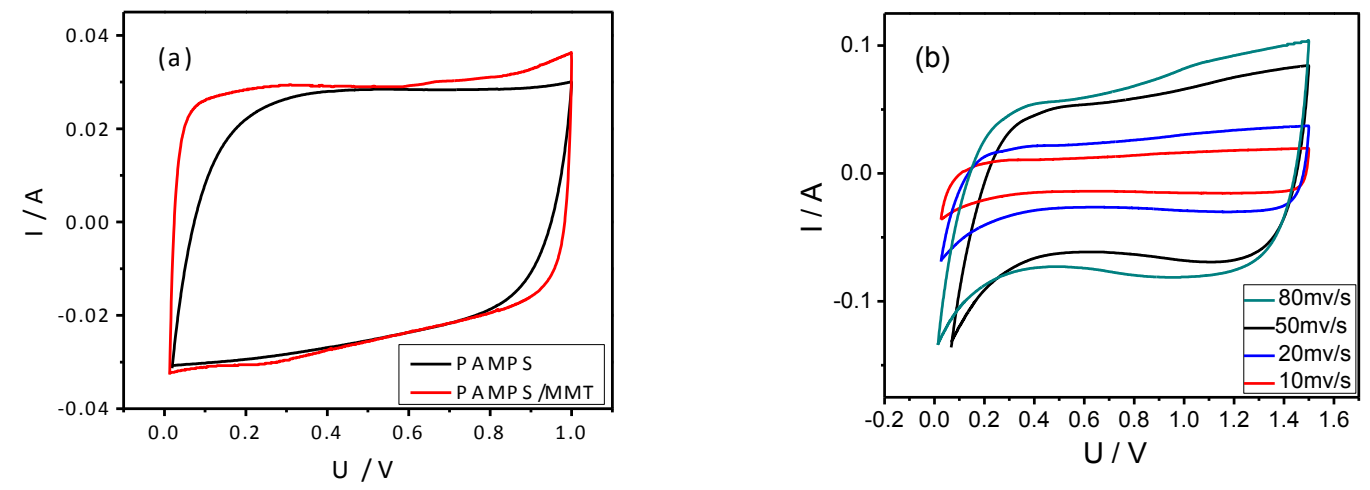

Fig.7 (a) CV plots of the EC based on PAMPS and PAMPS/MMT GPE, (b) CV plots of the EC based on PAMPS/MMT GPE at different scan rates
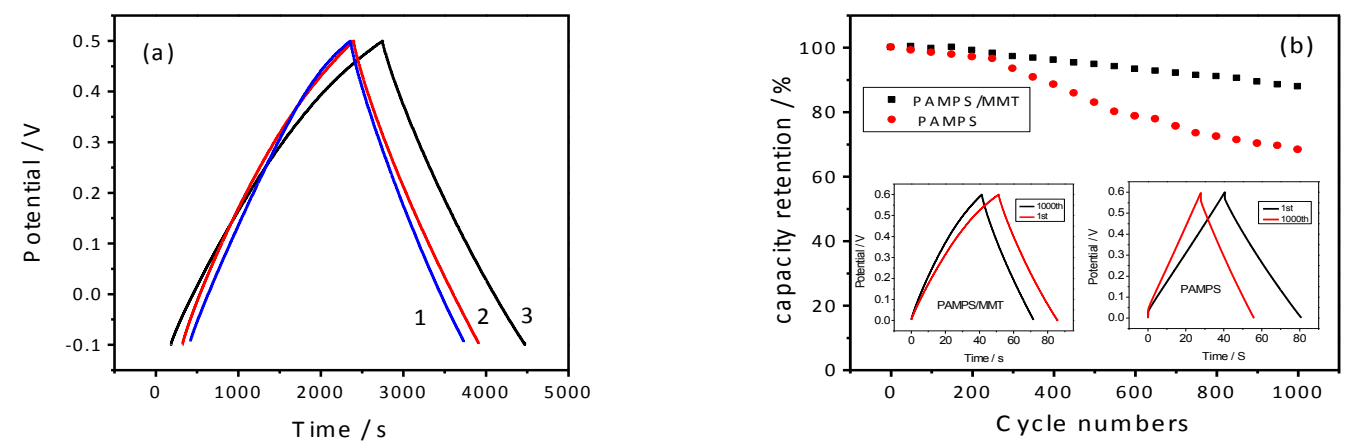

Fig.8 (a) Galvanostatic charge-discharge curves of the EC with (1) PAMPS GPE,

(2)PAMPS/MMT GPE and (3) 4M KOH aqueous solution electrolyte; (b) cycling performance of ECs based on PAMPS and PAMPS/MMT GPE at a current density of $0.2 \mathrm{~A} \mathrm{~g}^{-1}$ 
Table 1 Tensile strength of the PAMPS/MMT composite hydrogel with different proportion of

\begin{tabular}{cc}
\multicolumn{2}{c}{ Na-MMT } \\
\hline Na-MMT (wt $\%)$ & Tensile strength $(\mathrm{MPa})$ \\
\hline 0 & 6.9 \\
$3 \%$ & 7.2 \\
$5 \%$ & 8.3 \\
$7 \%$ & 8.9 \\
$9 \%$ & 7.9 \\
$11 \%$ & 6.3 \\
\hline
\end{tabular}

Table 2 Ionic conductivity of the PAMPS/MMT composite electrolyte with different proportion of Na-MMT

\begin{tabular}{cl}
\hline Na-MMT $(\mathrm{wt} \%)$ & $\sigma(\mathrm{S} / \mathrm{cm})$ \\
\hline 0 & $6.30 \times 10^{-3}$ \\
$3 \%$ & $2.87 \times 10^{-2}$ \\
$5 \%$ & $5.91 \times 10^{-2}$ \\
$7 \%$ & $4.83 \times 10^{-2}$ \\
$9 \%$ & $4.23 \times 10^{-2}$ \\
$11 \%$ & $2.86 \times 10^{-2}$ \\
\hline
\end{tabular}


Supplementary Information

\title{
PAMPS/MMT composite hydrogel electrolyte for solid-state supercapacitors
}

\author{
Jin Wang* ${ }^{\text {a }}$, Xianghu Yu ${ }^{\mathrm{a}}$, Cui Wang ${ }^{\mathrm{a}}$, Kechuang Xiang ${ }^{\mathrm{a}}$, Mengde Deng ${ }^{\mathrm{a}}$, and \\ Huabing Yin $^{\mathrm{b}}$ \\ ${ }^{a}$ School of Chemistry and Chemical engineering, Hefei University of Technology, Hefei 23009, P. \\ R. China. E-mail: jinwang@hfut.edu.cn \\ ${ }^{\mathrm{b}}$ Department of Electronics and Electrical Engineering, University of Glasgow, G12 8QQ, UK. \\ *Corresponding author Tel.: +86 551 62905660; fax: +86 55162905660 . \\ E-mail address: jinwang@hfut.edu.cn (Jin Wang).
}

\section{Contents:}

1. Swelling capacity and ionic conductivity of PAMPS hydrogel as a function of KPS loading weights (Fig. S1)

2. Swelling capacity and ionic conductivity of PAMPS hydrogel as a function of MBA loading weights (Fig. S2)

3. Tensile strength of PAMPS hydrogel as a function of MBA loading weights (Fig. S3) 


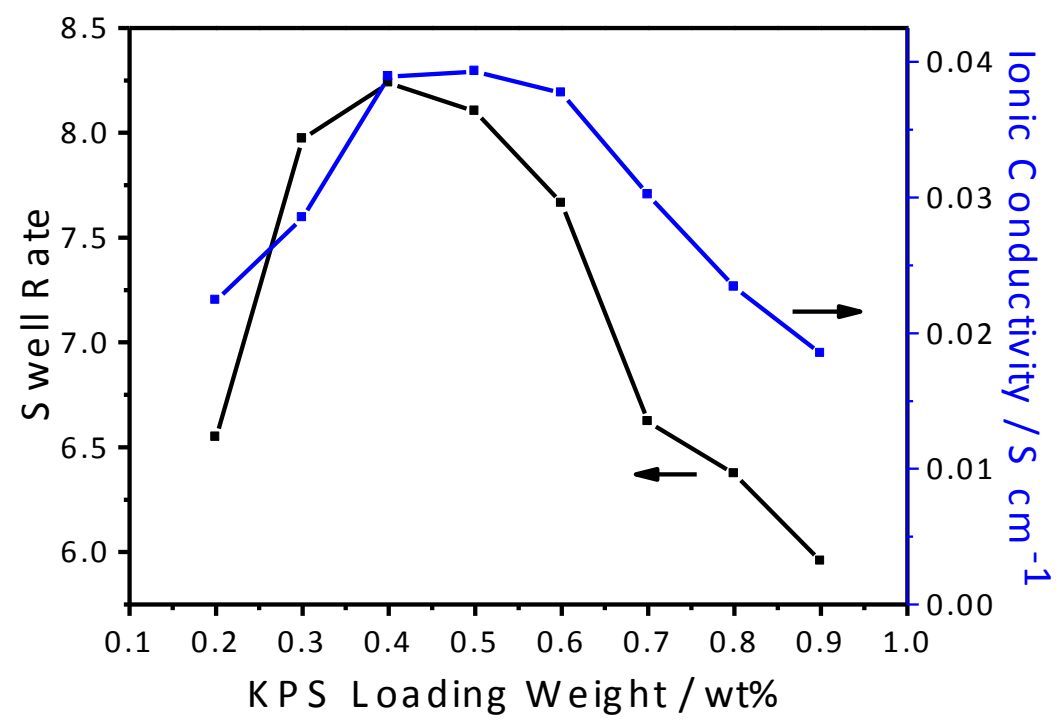

Figure S1. Swelling capacity and ionic conductivity of PAMPS hydrogel as a function of KPS loading weights

As shown in Figure S1., both the swelling capacity and the ionic conductivities of PAMPS hydrogel were all increased with increasing the content of KPS first, and then decreased with load increasing. It should be due to the molecular weight of PAMPS is increased with increasing the content of KPS when KPS in a small amount, and the larger macromolecule has more swell capacity which is contributed to the ionic conductivity of PAMPS hydrogel. As the consequential KPS load increasing, the capacity of polymerization would be increased for the higher concentration of free radicals, which results in the decreasing of molecular weight of PAMPS. The lower molecular weight of PAMPS has the lower swelling capacity and so decreased the ionic conductivity of PAMPS hydrogel. 


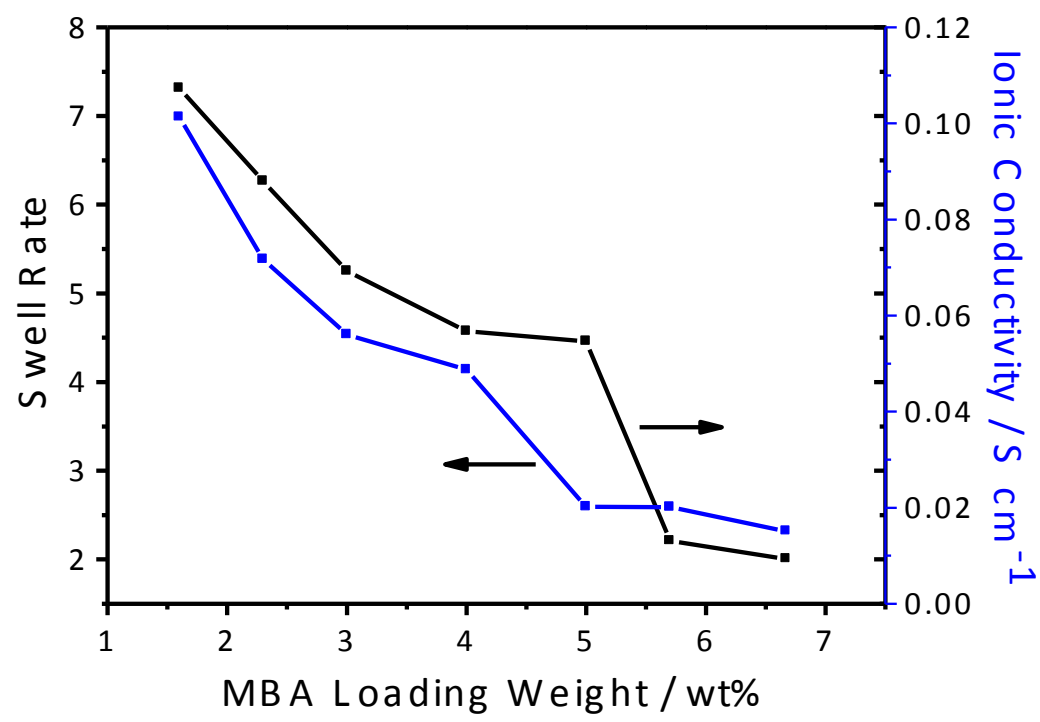

Figure S2. Swelling capacity and Ionic conductivity of PAMPS hydrogel as a function of MBA loading weights

As shown in Figure S2., both the swelling capacity and the ionic conductivities of PAMPS hydrogel were all decreased with increasing the content of MBA. The cross-linking density is increased when increasing the content of cross-linking agent, and hindering the movement of chain segment of PAMPS. So the swelling capacity is decreased and the ionic conductivities of PAMPS hydrogel were decreased accordingly. 


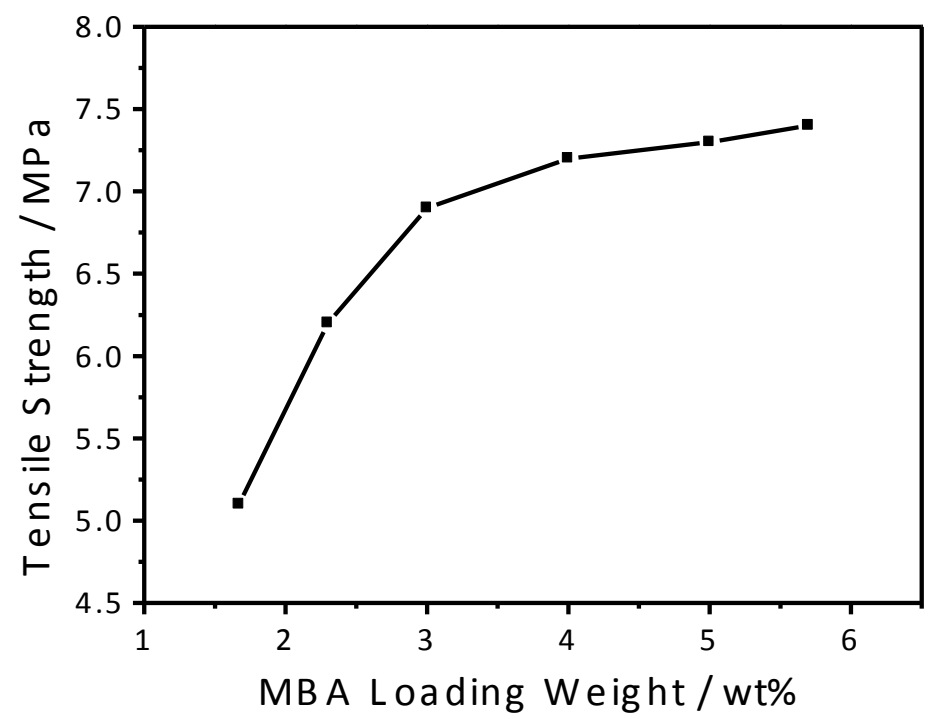

Figure S3. Tensile strength of PAMPS hydrogel as a function of MBA loading weights

As shown in Figure S3., the tensile strength of PAMPS hydrogel is increased with increasing the contents of MBA. The linear molecule of PAMPS was transfer into the three dimensional network with the addition of cross-linking agent, and the enhanced cohesion strength of PAMPS is resist the relative slippage of the molecular chain when stretch PAMPS hydrogel. 